\title{
SOME RESULTS ON THE RADIAL PROJECTION IN BANACH SPACES
}

\author{
R. L. THELE ${ }^{1}$
}

Abstract. We obtain in this paper some new theorems on the radial projection onto the unit ball in Banach spaces, including in particular the following: A Banach space $X$ is uniformly nonsquare if and only if the radial projection in $X$ has Lipschitz constant strictly less than 2 .

In a real normed linear space $X$ the mapping $T: X \rightarrow X$ defined by

$$
\begin{aligned}
T x & =x, & & \|x\| \\
& =x /\|x\|, & \|x\| & \geqq 1,
\end{aligned}
$$

is called the radial projection of $X$ onto the unit ball in $X$. In this paper we investigate first the relationship between the least Lipschitz constant $k(X)$ of $T$ and the concept of orthogonality of R. C. James [4]. (Recall that a vector $x \in X$ is said to be orthogonal to $y \in X$ (denoted $x \perp y$ ) if $\|x\| \leqq\|x-\lambda y\|$ for every real $\lambda$.) D. deFigueiredo and L. Karlovitz initiated such a study in [1] by proving that the orthogonality relation is symmetric if and only if $k(X)=1$. We prove that, in fact,

$$
k(X)=\sup \{\|y\| /\|y-\lambda x\|: y \neq 0, x \perp y, \lambda \in R\}
$$

where $R$ denotes the field of real numbers. This characterization enables us to obtain as an immediate consequence the theorem of deFigueiredo and Karlovitz, and permits us to obtain our second main result, in which we characterize uniformly nonsquare Banach spaces as follows: $k(X)=2$ if and only if $X$ is not uniformly nonsquare.

We might remark that it is generally known (cf. [2]) that the constant $k(X)$ always satisfies $1 \leqq k(X) \leqq 2$.

We begin with the following:

LEMmA. The constant $k=k(X)$ satisfies

(i) $k=\sup \{\|x-y /\| y\|\| /\|x-y\|: x, y \in X,\|x\| \leqq 1<\|y\|\}$

(ii) $k=\sup \{\|u-v\| /\|u-\lambda v\|\}: u, v \in X,\|u\| \leqq\|v\|=1, \lambda>1\}$.

Received by the editors October 6, 1972 and, in revised form, March 26, 1973.

AMS (MOS) subject classifications (1970). Primary 46B99.

1 Theorem 1 is taken from the author's University of Iowa doctoral dissertation, written under the supervision of Professor W. A. Kirk.

(C) American Mathematical Society 1974 
Proof. Let

$$
m=\sup \{\|x-y /\| y\|\| /\|x-y\|: x, y \in X,\|x\| \leqq 1<\|y\|\} .
$$

Clearly, $m \leqq k$. To show $m=k$ we show that $m$ is a Lipschitz constant for $T$, and to do this we need only verify that $\|x /\| x\|-y /\| y\|\| \leqq m\|x-y\|$ whenever $1 \leqq\|x\|<\|y\|$. Now, letting $x^{\prime}=x /\|x\|$ and $y^{\prime}=y /\|x\|$, we have $\left\|x^{\prime}\right\|=1<\left\|y^{\prime}\right\|$, and thus

$$
\begin{aligned}
\left\|\frac{x}{\|x\|}-\frac{y}{\|y\|}\right\| & =\left\|x^{\prime}-\frac{y^{\prime}}{\left\|y^{\prime}\right\|}\right\| \\
& \leqq m\left\|x^{\prime}-y^{\prime}\right\| \leqq m\|x-y\|
\end{aligned}
$$

whenever $1 \leqq\|x\|<\|y\|$.

The second statement follows if in (i) we set $x=u$ and $y=\lambda v$, with $\|v\|=1$ and $\lambda>1$.

We remark that if $x, y \in X$ are distinct, $y \neq 0$, then the function $f: R \rightarrow R$ defined by $f(\lambda)=\|x-\lambda y\|$ is convex; moreover, there exists a closed interval $\left[\lambda_{1}, \lambda_{2}\right]$ on which $f$ attains its minimum and such that $f$ is strictly increasing on $\left[\lambda_{23} \infty\right)$ and strictly decreasing on $\left(-\infty, \lambda_{1}\right]$. We note that $\lambda_{1}=\lambda_{2}$ if $X$ is strictly convex.

We observe further that if $\lambda_{0}$ minimizes $\|x-\lambda y\|$, then $\left(x-\lambda_{0} y\right) \perp y$.

TheOREM 1. $k(X)=\sup \{\|y\| /\|y-\lambda x\|: y \neq 0, x \perp y, \lambda \in R\}$.

Proof. From the lemma

$$
\begin{aligned}
k(X) & =\sup \left\{\frac{\|u-v\|}{\|(u-v)-\mu v\|}:\|u\| \leqq\|v\|=1, \mu>0\right\} \\
& =\sup \left\{\frac{\|y\|}{\|y-\mu v\|}:\|v+y\| \leqq\|v\|, v \neq 0, \mu>0\right\}
\end{aligned}
$$

where $u-v=y$. Suppose $x \perp y, y \neq 0$, and $\lambda \in R$. Then $\|(\lambda / \mu) x\| \leqq$ $\|(\lambda / \mu) x-y\|$, so that letting $v=(\lambda / \mu) x-y$ we obtain

$$
\begin{aligned}
k(X) & \geqq \sup \left\{\frac{\|y\|}{\|y-\mu[(\lambda x / \mu)-y]\|}: x \perp y, y \neq 0, \lambda \in R, \mu>0\right\} \\
& =\sup \left\{\frac{\|y\|}{\|(1+\mu) y-\lambda x\|}: x \perp y, y \neq 0, \lambda \in R, \mu>0\right\} \\
& =\sup \left\{\frac{\|y\|}{\|y-\lambda x\|}: x \perp y, y \neq 0, \lambda \in R\right\} .
\end{aligned}
$$

Now let $y, v$, and $\mu$ be as in (1). Since

$$
\|(y-\mu v)-(\mu+1) y\|=\mu\|v+y\| \leqq \mu\|v\|=\|(y-\mu v)-y\|,
$$


the minimum of $\|(y-\mu v)-\lambda y\|$ is attained for some $\lambda_{0}>1$. Then $x \perp y$ if $x=(y-u v)-\lambda_{0} y$. Also,

so that

$$
\frac{\|y\|}{\|y-\mu v\|}=\frac{\|y\|}{\left\|\lambda_{0} y+x\right\|} \leqq \frac{\|y\|}{\left\|y+\lambda_{0}^{-1} x\right\|}
$$

$$
k(X) \leqq \sup \left\{\frac{\|y\|}{\|y-\lambda x\|}: x \perp y, y \neq 0, \lambda \in R\right\}
$$

Corollary (DeFigueiredo AND Karlovitz [1]). In a real normed space $X, k(X)=1$ if and only if orthogonality is symmetric in $X$.

Proof. If $k(X)=1$, then whenever $x \perp y$ and $y \neq 0,\|y\| /\|y-\lambda x\| \leqq 1$ for every $\lambda \in R$, and thus $y \perp x$. On the other hand, if orthogonality is symmetric in $X$, then whenever $x \perp y,\|y\| \leqq\|y-\lambda x\|$ for every $\lambda \in R$, and thus $k(X) \leqq 1$.

A Banach space $X$ is said to be uniformly nonsquare [5] if there exists $\delta>0$ such that whenever $\|x\|=\|y\|=1$ then either $\|(x+y) / 2\| \leqq 1-\delta$ or $\|(x-y) / 2\| \leqq 1-\delta$. It is easily verified (cf. [3]) that every uniformly convex space is uniformly nonsquare.

THEOREM 2. A Banach space $X$ is uniformly nonsquare if and only if $k(X)<2$.

Proof. Suppose $k(X)=2$. For arbitrary $\delta>0$, choose $\varepsilon>0$ such that

$$
(1-\varepsilon) /(1+2 \varepsilon)>1-\delta .
$$

From Theorem 1 there exist $x$ and $y$ such that $x \perp y,\|x\|=\|y\|=1$, and there exists $\lambda>0$ such that $\|2 y-\lambda x\|<1+\varepsilon$. Then $1-\varepsilon<\lambda<1+\varepsilon$, since $\|x\|=\|y\|=1$ and $x \perp y$. Thus

$$
1 \leqq\|2 y-x\|<1+2 \varepsilon .
$$

Let $u=x$ and $v=(2 y-x) /\|2 y-x\|$. Then $\|u\|=\|v\|=1$, and

$$
\begin{aligned}
\left\|\frac{1}{2}(u+v)\right\| & =\left\|\frac{y-\frac{1}{2}(1-\|2 y-x\|) x}{\|2 y-x\|}\right\|>\frac{1-\varepsilon}{1+2 \varepsilon}>1-\delta, \\
\left\|\frac{1}{2}(u-v)\right\| & =\left\|\frac{y-\frac{1}{2}(1+\|2 y-x\|) x}{\|2 y-x\|}\right\| \\
& \geqq \frac{\frac{1}{2}(1+\|2 y-x\|)}{1+2 \varepsilon} \geqq \frac{1}{1+2 \varepsilon}>1-\delta,
\end{aligned}
$$

which implies that $X$ is not uniformly nonsquare. 
Conversely, if $X$ is not uniformly nonsquare, then for any $\delta>0$ there exist $x$ and $y$ such that $\|x\|=\|y\|=1$ and

$$
\left\|\frac{1}{2}(x+y)\right\|>1-\delta, \quad\left\|\frac{1}{2}(x-y)\right\|>1-\delta .
$$

For arbitrary $\mu>\lambda>1$ choose $\delta>0$ such that $\delta<(\mu-\lambda) / 2 \mu$. Now we apply (ii) of the lemma with $u=\lambda x$ and $v=x+(\mu-1) y$. Then $\|u\|=\lambda$ and

$$
\begin{aligned}
\|v\| & =\|\mu(x+y)-(\mu-1) x-y\|>2 \mu(1-\delta)-(\mu-1)-1 \\
& =\mu(1-2 \delta)>\lambda,
\end{aligned}
$$

so that $\|v\|>\|u\|$. Also,

$$
\begin{aligned}
\frac{\|u-v\|}{\|u-\lambda v\|} & =\frac{\|(\lambda-1)(x-y)-(\mu-\lambda) y\|}{\|\hat{\lambda}(\mu-1) y\|} \\
& \geqq \frac{2(\lambda-1)(1-\delta)-(\mu-\lambda)}{\lambda(\mu-1)} \geqq \frac{(\lambda-1)(\mu+\lambda)-\mu(\mu-\lambda)}{\mu \lambda(\mu-1)} .
\end{aligned}
$$

Letting first $\lambda \rightarrow \mu$ and then $\mu \rightarrow 1$, we see that $k(X)=2$.

The author wishes to express his gratitude to the referee for simplifying some of the original arguments.

\section{REFERENCES}

1. D. G. deFigueiredo and L. A. Karlovitz, On the radial projection in normed spaces, Bull. Amer. Math. Soc. 73 (1967), 364-368. MR 35 \#2130.

2. C. F. Dunkl and K. S. Williams, A simple norm inequality, Amer. Math. Monthly 71 (1964), 53-54.

3. K. Goebel, Convexity of balls and fixed-point theorems for mappings with nonexpansive square, Compositio Math. 22 (1970), 269-274. MR 42 \#8355.

4. R. C. James, Orthogonality and linear functionals in normed linear spaces, Trans. Amer. Math. Soc. 61 (1947), 265-292. MR 9, 42.

5. — Uniformly non-square Banach spaces, Ann. of Math. (2) 80 (1964), 542550. MR $30 \# 4139$.

6. W. A. Kirk and M. F. Smiley, Another characterization of inner product, Amer. Math. Monthly 71 (1964), 890-891.

Department of Mathematics, University of Iowa, Iowa City, Iowa 52242

Current address: Department of Mathematics, University of Oklahoma, Norman, Oklahoma 73069 